\title{
MICROESCULTURA DA CONCHA DE RECTARTEMON (RECTARTEMON) DEPRESSUS (HEYNEMANN) (GASTROPODA, STYLOMATOPHORA, STREPTAXIDAE)
}

\author{
Mônica Picoral ${ }^{1,2}$ \\ Vera Lúcia Lopes-Pitoni ${ }^{1}$
}

\begin{abstract}
THE MiCROSCULPTURE OF THE SHELl OF RECTARTEMON (RECTARTEMON) DEPRESSUS (HEYNEMANN) (GASTROPODA, Stylomatophora, StreptaXidAE). The microsculpture of Rectartemon depressus (Heynemann, 1868) shell is described on scanning electron microscope. The generics characteristics are proposed: shell's dorsal sculture formed for ribs striae radial; nuclear whorls smooth; ventral surface of the shell smooth or slightly striate; and as specifics characteristics: the number of ribs striae radial on the last world. The $R$. (R.) depressus presented seven or eight ribs striae radial for millimeter.

KEY WORDS. Stylommatophora, Streptaxidae, Rectartemon (Rectartemon) depressus, photomicrography, shell
\end{abstract}

Segundo BLANFORD \& GodWIN-AUsten (1908) e BRUGgen (1967) a família Streptaxidae está amplamente distribuída nas regiões tropical e subtropical do mundo, sendo que a maioria das espécies descritas ocorrem na África e América do Sul.

PARKINSON et al. (1987) comentam que esta família compõe o grupo carnivoro dominante na sub-região Brasiliana, sendo que os gêneros Rectartemon Baker, 1925, Streptaxis Gray, 1837, Streptartemon Kobelt, 1905 e Martinella Jousseaume, 1887 também habitam a sub-região Colombiana.

Há registros de Rectartemon (Rectartemon) depressus (Heynemann, 1868) no Rio Grande do Sul, para Taquara (KoBELT 1905), para Morro Reuter (= Rödersberg/Costa da Serra) (MARTENS 1868) e para Guaíba (HEYDRICH \& THOMÉ 1990). Os dois primeiros trabalhos restringem-se à morfologia da concha e dados conquiliométricos. No terceiro, os autores fazem a recaracterização conquiliológica de $R$. (R.) depressus. O sistema reprodutor é descrito por PICORAL \& THOMÉ (1993, 1996).

Com a finalidade de contribuir para a diagnose do gênero Rectartemon, analisa-se a fotomicrografia de varredura da concha de $R$. (R.) depressus.

1) Museu de Ciências Naturais, Fundação Zoobotânica do Rio Grande do Sul. Avenida Salvador França 1427, 90690-000 Porto Alegre, Rio Grande do Sul, Brasil.

2) Bolsista CNPq/RHAE. 


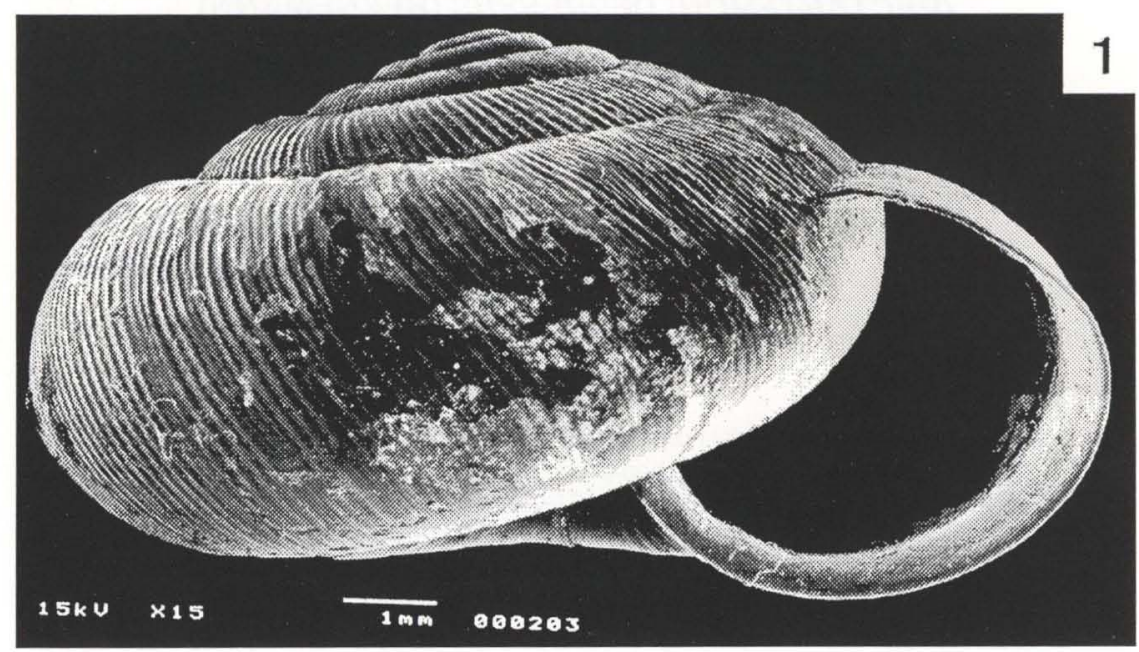

Fig. 1. Fotomicrografia de varredura da concha de Rectartemon (Rectartemon) depressus, vista frontal. (MCN n³2514).

\section{MATERIAL E MÉTODOS}

Os exemplares de $R$. (R.) depressus fazem parte do lote $n^{\circ} 32514$ da coleção malacológica do Museu de Ciências Naturais da Fundação Zoobotânica do Rio Grande do Sul (MCN/FZB), coletados no município de Guaíba/Rio Grande do Sul, km 307 da BR 116 (PICORAL \& THOMÉ 1993; MATZENBACHER 1985).

Foram estudadas oito conchas de espécimes adultos, limpas com ultra-som ou em bateria alcoólica crescente $(70 \%, 80 \%, 90 \%, 96 \%$ e $100 \%)$ por aproximadamente $15 \mathrm{~min}$, deixadas secar naturalmente por dois dias; montadas sobre "stubs" com fita adesiva dupla face ou com esmalte de unha; metalizadas com ouro e fotografadas ao microscópio eletrônico de varredura (MEV) Jeol JSM 5200 do (MCN/FZB).

Comparações, em observação ao estereomicroscópio, foram realizadas com material incluído na coleção da Academy of Natural Sciences of Philadelphia (ANSP), Estados Unidos da América do Norte: Artemon capillosus (Pilsbry, 1897) (ANSP n²3758/tipo, Bahia/Brasil, Swift Colln.); A. decussatus (Pilsbry, 1897) (ANSP n 23764/holotipo, Brasil, J.S. Phillips); A. helios (Pilsbry, 1897) (ANSP n ${ }^{\circ}$ 23765/tipo, Brasil, Swift Coll, Moricand); A. tumulus (Pilsbry, 1897) (ANSP n ${ }^{\circ}$ 23766/tipo, Brasil, J.S. Phillips), A. iguapensis Pilsbry, 1930 (ANSP nº 152245/tipo, Iguapé/São Paulo/Brasil, H. von Ihering) e A. iheringi Pilsbry, 1930 (ANSP nº 151896/tipo, Brasil, H. von Ihering). 


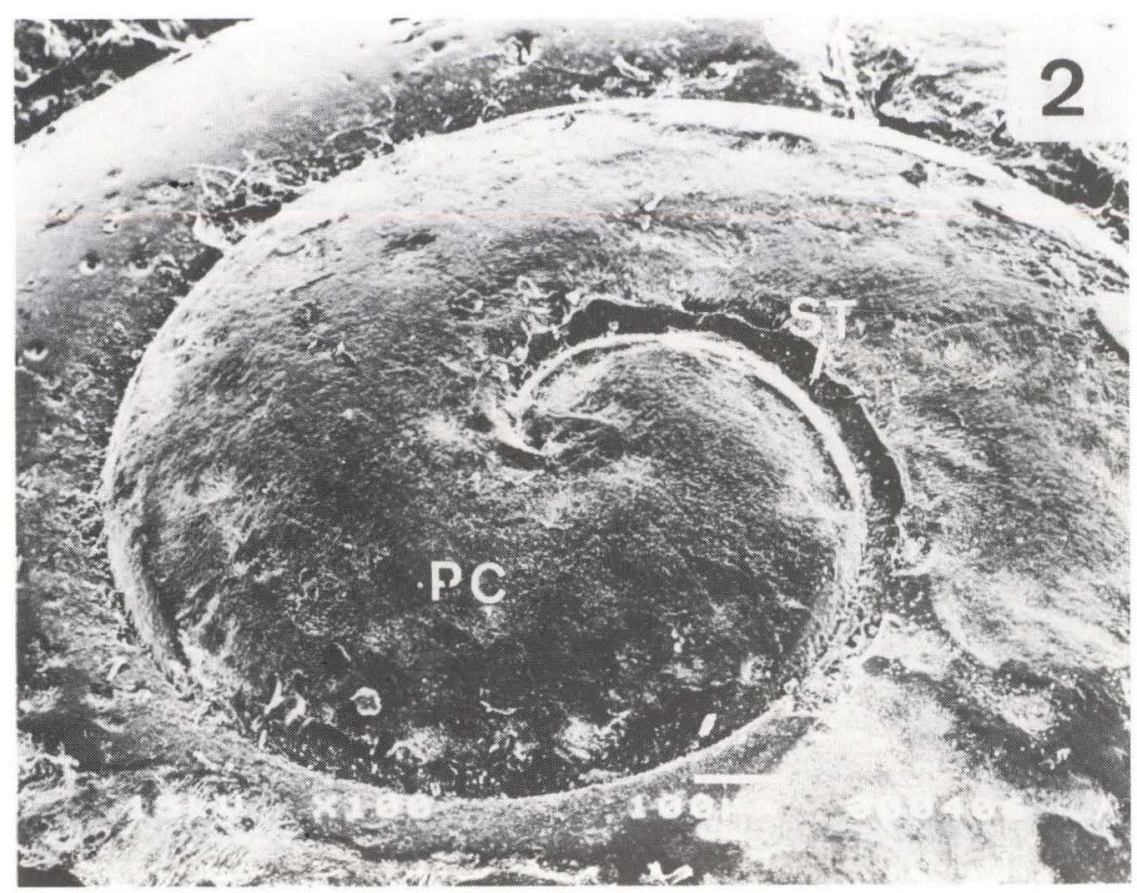

Fig. 2. Fotomicrografia de varredura da concha de Rectartemon (Rectartemon) depressus, protoconcha, vista dorsal. (PC) Protoconcha, (TC) teleoconcha; (ST) sutura; (ES) estrias costeladas.

\section{RESULTADOS}

A concha de Rectartemon (Rectartemon) depressus caracteriza-se pela forma globosa-conóide (Fig. 1), com a protoconcha lisa (Fig. 2), constituída por 2,25 a 2,5 voltas iniciais apresentando limite bem demarcado com a teleoconcha (Fig. 3). As voltas embrionárias são bem destacadas da espira, em vista frontal (Figs 1, 4).

A teleoconcha constitui as 3 a 3,5 voltas finais da concha, apresentando sutura profunda e acanalada. A esculturação é formada por estrias costeladas radiais, e linhas de crescimento. Não há microescultura ao MEV.

As estrias costeladas (Figs 1, 4-6) no início das voltas pós-embrionárias são pouco demarcadas e mais largamente espassadas, tornando-se a partir da terceira volta mais conspícuas e próximas. Na última volta contam-se sete a oito estrias costeladas por milímetro ( $7 \mathrm{a} 8 / \mathrm{mm})$, que se estendem desde a sutura até a superfície ventral onde são vestigiais junto a base (Figs 1,7,8).

As linhas de crescimento são representadas por estrias-sulcadas nas superfícies dorsal e ventral (Figs 5, 6, 8). Na última volta, em vista ventral, podem apresentarem-se como costelas (Fig. 7) tornando-se mais salientes próximo ao umbílico, que se apresenta amplo e perspectivo (Figs 7, 8). 


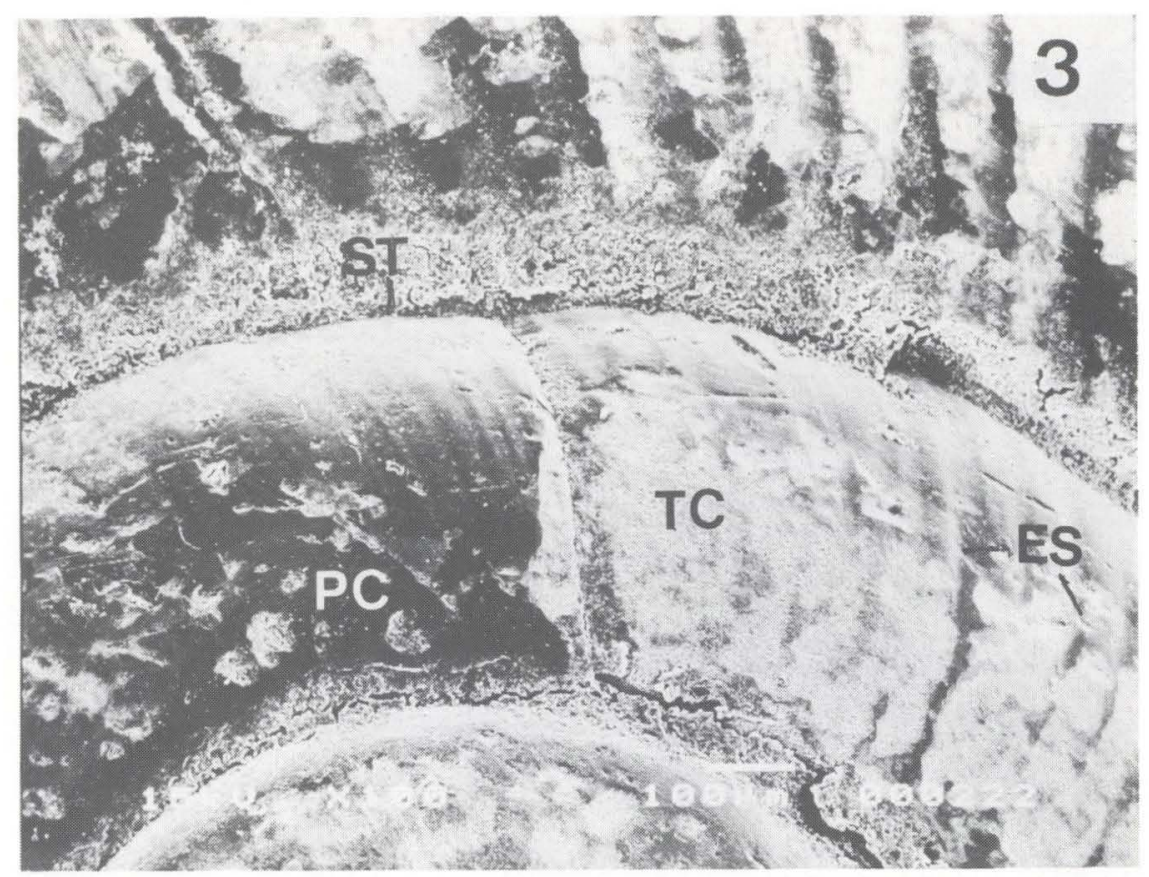

Fig. 3. Fotomicrografia de varredura da concha de Rectartemon (Rectartemon) depressus, detalhe da protoconcha/teleoconcha, vista dorsal. (PC) Protoconcha, (TC) teleoconcha; (ST) sutura; (ES) estrias costeladas.

\section{DISCUSSÃO}

A presença de estrias costeladas na superfície dorsal da concha, a ausência de esculturações na superfície ventral tornando a base lisa ou com estrias brevemente marcadas e a protoconcha lisa variando em torno de 2,5 a 3 voltas iniciais, são descritas para Artemon capillosus (Pilsbry, 1897), A. tumulus (Pilsbry, 1897), A. iguapensis Pilsbry, 1930, A.iheringi Pilsbry, 1930, A. intermedius piquetensis Pilsbry, 1930, e A. colombianus Pilsbry, 1935 (PILSBRy 1897, 1930, 1935), A. muelleri Thiele, 1927 (THIELE 1927). As esculturações dorsal e ventral da concha e a protoconcha lisa conferem com a descrição feita para a concha de $R$. (R.) depressus.

Para A. wagneri (Pfeiffer, 1841), A. regius (Löbbecke, 1881), A. politus (Fulton, 1899), A. intermedius (Albers, 1857), os autores descrevem a concha com esculturações dorsais apresentando costelas oblíquas densamente esculpidas e ventralmente lisa, não descrevendo detalhes da protoconcha (ALBERS 1857; FULTON 1899; LÖBBECKE 1881; PFEIFFER 1846). Estas espécies não apresentam dados suficientes para uma comparação mais precisa.

PILSBRY (1897) descreve a concha da espécie nova A. helios com estrias dorsais brevemente marcadas próximas à sutura. Este tipo de esculturação, analisada ao estereomicroscópio, difere das espécies citadas, diferindo também de $R$. (R.) depressus, em análise ao estereomicroscópio e ao MEV. 


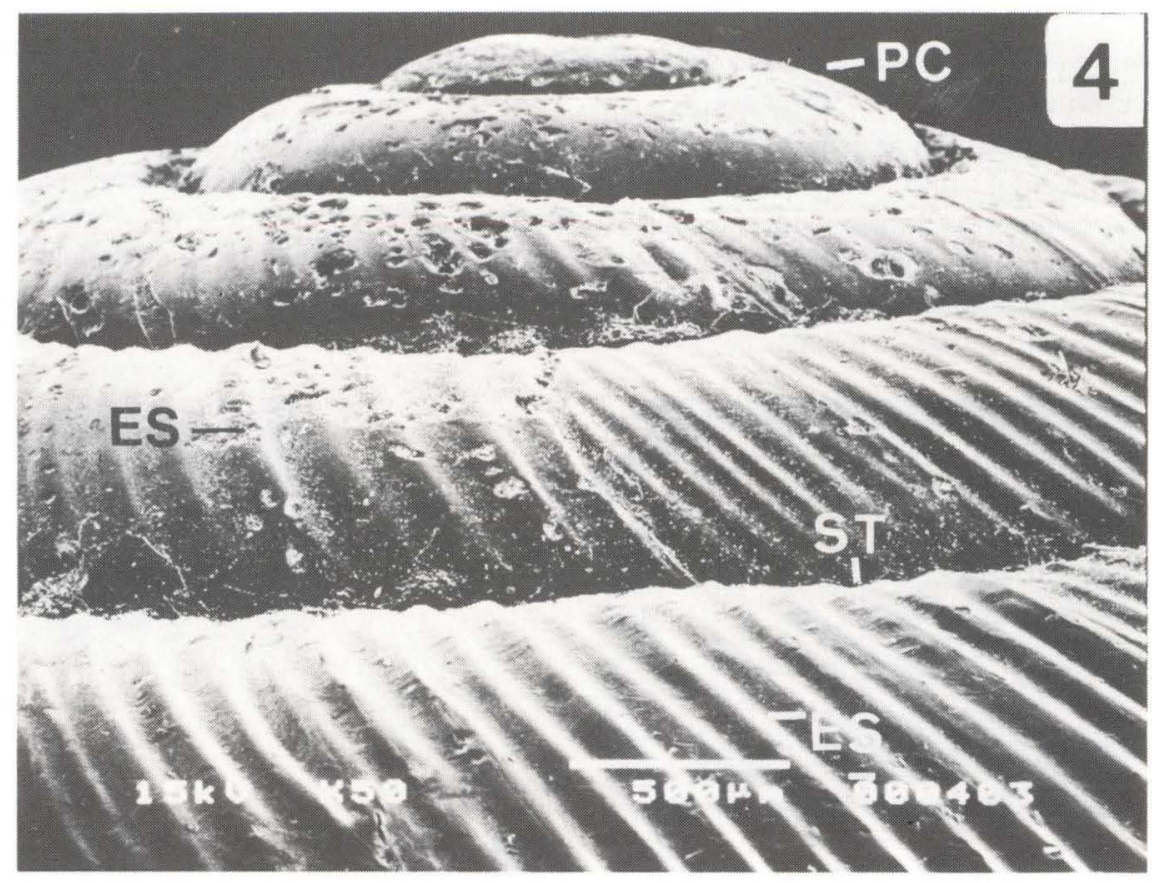

Fig. 4. Fotomicrografia de varredura da concha de Rectartemon (Rectartemon) depressus, espira, vista frontal. (PC) Protoconcha, (TC) teleoconcha; (ST) sutura; (ES) estrias costeladas.

BAKER (1925) descreve a espécie-tipo Rectartemon jessei Baker, 1925, referindo-se à esculturação como linhas de crescimento salientes mais estreita que seus intervalos $(3 \mathrm{~mm})$; base lisa e, protoconcha lisa em torno de 2,75 voltas iniciais. As características da esculturação coincidem com àquelas de $R$. (R.) depressus, diferindo apenas no número de estrias costeladas por milímetro $(3 / \mathrm{mm})$, que acredita-se que o autor refere-se como linhas de crescimento.

HEYDRICH \& THOMÉ (1990) descrevem a concha de $R$. (R.) depressus sob o estereomicroscópio, porém os autores caracterizam as esculturas dorsais como linhas de crescimento costeladas em torno de 6 a 10 por milímetro $(6$ a $10 / \mathrm{mm})$ na última volta e labros de crescimento, e a escultura ventral, lisa com labros de crescimento. Acredita-se que as linhas de crescimento costeladas sejam o que denominou-se de estrias costeladas e os labros de crescimento sejam as formações sulcadas (dorsalmente) e costelares (ventralmente), formas de apresentação denominadas para as linhas de crescimento. O número de estrias costeladas ( $7 \mathrm{a} 8 / \mathrm{mm})$ analisadas ao MEV difere da contagem, realizada pelos autores, ao estereomicroscópio.

A presença de um umbílico amplo e perspectivo, que é perfeitamente visível ao estereomicroscópio, é descrito para todas as espécies aqui mencionadas. Analisando as conchas das espécies $A$. capillosus, A. helios, A. tumulus, A. iguapensis, $A$. iheringi, constatou-se a presença de linhas de crescimento com aspecto de 
formações costelares na base da concha, próximas ao umbílico, semelhante às descritas para $R$. (R.) depressus.

O número de estrias costeladas na última volta, analisadas ao estereomicroscópio, em A. capillosus é de cinco por milímetro ( $5 / \mathrm{mm})$, em A. decussatus é de três por milímetro $(3 / \mathrm{mm})$ e em $A$. tumulus é de dois e meio por milímetro $(2,5 / \mathrm{mm})$. Estes números diferem de $R$. (R.) depressus onde conta-se, ao estereomicroscópio, em torno de dez a doze estrias costeladas por milímetro (10 a $12 / \mathrm{mm})$ e ao MEV, sete a oito estrias costeladas por milímetro $(7 \mathrm{a} 8 / \mathrm{mm})$ na última volta.
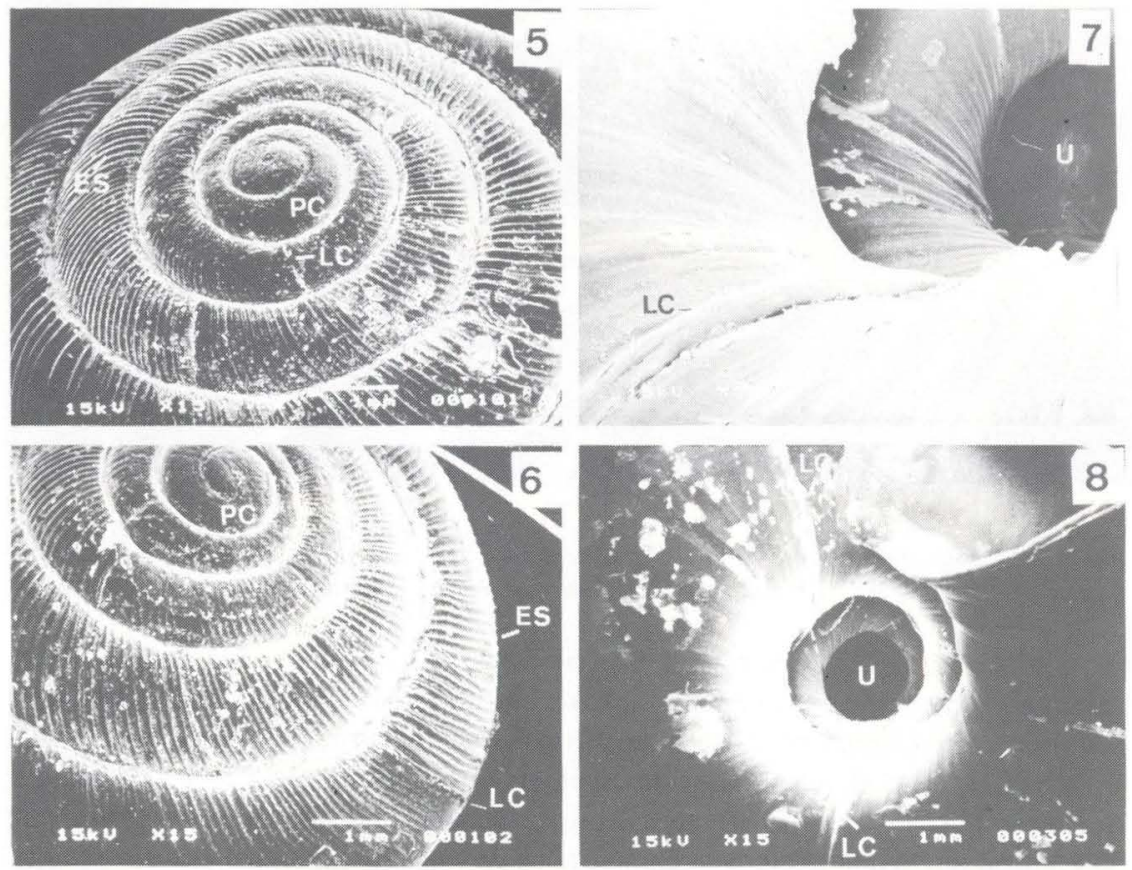

Figs 5-8. Fotomicrografia de varredura da concha de Rectartemon (Rectartemon) depressus. (5-6) Vista dorsal; (7-8) vista ventral. (ES) Estrias costeladas, (LC) linhas de crescimento, (PC) protoconcha, (U) umbilico.

\section{CONCLUSÕES}

Acredita-se que à nível de microscopia eletrônica de varredura possa haver diferenciações na esculturação da concha das espécies do gênero Rectartemon. Estas diferenciações seriam mais evidentes, principalmente no que se refere ao número de estrias costeladas por milímetro.

Propõe-se como características descritivas utilizáveis à nível genérico (Rectartemon): 1) esculturações dorsais da concha formadas por estrias costeladas radiais; 2) protoconcha lisa; 3) superfície ventral da concha lisa ou brevemente estriada. 
Características à nível específico o número, por milímetro, de estrias costeladas radiais presentes na última volta da concha.

Em Rectartemon (R.) depressus: 4) sete a oito estrias costeladas radiais por milímetro.

AGRADECIMENTOS. Ao Programa RHAE/CNPq; ao técnico Cleodir J. Manssan, do Laboratório de Microscopia Eletrônica de Varredura do $\mathrm{MCN} / \mathrm{FZB}$, pelo auxílio e sugestões; à Academy of Natural Sciences of Philadelphia (ANSP), Estados Unidos da América do Norte, pelo empréstimo do material científico malacológico.

\section{REFERÊNCIAS BIBLIOGRÁFICAS}

AlBERS, J.C. 1857. Diagnosen neuer Heliceen mit gelegentlicher Berichtigung einiger älteren Arten. Malak. Bl., Cassel, 4: 89-100.

BAKER, H.B. 1925. The mollusca collected by the University of Michigan-Williamson Expedition in Venezuela. Part III. Pupilidae to Oleacinidae. Occ. Pap. Mus. Zool. Univ. Mich., Ann Arbor, 156: 1-56.

Blanford, W.T. \& H.H. Godwin-Austen. 1908. Mollusca Testacellidae and Zonitidae, p.1-24. In: C.T. BINGHAM (Ed.). The fauna of British India including Ceylon and Burma.

Bruggen, A.C. 1967. An Introduction to the Pulmonate family Streptaxidae Jou. Conch. Lond., London, 26 (3): 181-188.

Fulton, H. 1899. Descriptions of supposed new species of Streptaxis and Amphidromus. Proc. malac. Soc. Lond., London, 3 (6): 302-303.

Heydrich, I. \& J.W. ThOMÉ. 1990. Padronização de características conquiliológicas aplicáveis aos Streptaxidae sulbrasileiros (Mollusca; Gastropoda), p.536537. In: Anais da Sociedade para o Progresso da Ciência. São Paulo, vol. 42, $567 \mathrm{p}$.

KoBELT, W. 1905. Die Raublungenschnecken (Agnatha). Zweite Abteilung: Streptaxidae und Daudebardiidae. In: F.H.W. MARTINI \& J.H. ChEMNITZ. Systematisches Conchylien-Cabinet, Nurenberg, 1: 1-211p.

LöBBEcke, T. 1881. Diagnosen neuer Arten. Nachr. Bl. dt. malakozool. Ges., Frankfurt, 13 (4): 49-51.

MARTENS, E. 1868. Über südbrasilische Land-und Süsswasser Mollusken nach den Sammlungen von Dr. R. Hensel. Malak. Bl., Cassel, 15: 169-217.

MATZENBACHER, N.I. 1985. Levantamento florístico preliminar das compostas da fazenda São Maximiano - Guaíba - RS - Brasil. Comun. Mus. Ci. PUCRS, sér.Bot., Porto Alegre, 37: 115-127.

PARKINSON, B.; J. HEMMEN \& K. GROH. 1987. Tropical Landshells of the word. Wiesbaden, Western Germany, Verlag Christa Hemmen, 279p.

PFEIFFER, L. 1846. Die Schnirkelschnecken (Gattung Helix). In: F.H.W. MARTINI \& J.H. CHEMNITZ. Systematisches Conchylien-Cabinet, Nurenberg, 1: 1-400. PICORAL, M. \& J.W. ThOMÉ. 1993. Anatomia do sistema reprodutor de Rectartemon (Rectartemon) depressus (Heynemann, 1868) (Mollusca; Gastropoda; 
Streptaxidae). Biociências, Porto Alegre, 1 (1): 5-24.

. 1996. Anatomia e microanatomia do sistema reprodutor de Rectartemon (Rectartemon) depressus (Heynemann, 1868) (Mollusca, Gastropoda, Streptaxidae): Ovispermioducto ao Poro genital. Biociências, Porto Alegre, 4 (1): 103-119.

Pilsbry, H.A. 1897. New Brasilian Streptaxidae. Proc. Acad. nat. Sci. Philad., Philadelphia, 49: 477-479.

1930. South American land and freshwater mollusks: Notes and Descriptions - VII. Proc. Acad. nat. Sci. Philad., Philadelphia, 82: 355-365. 1935. South American land and freshwater mollusks, IX - Colombian species. Proc. Acad. nat. Sci. Philad., Philadelphia, 87: 83-88.

THIELE, J. 1927. Über einige brasilianische Landsnecken. Abh. sensckenb. naturforsch. Ges., Frankfurt, 40 (3): 307-329.

Recebido em 28.II.1996; aceito em 27.XI.1997. 